\title{
Highly Substituted Poly(2,3-diphenyl-1, 4-phenylenevinylene) Derivatives Having Bulky Phenyl and Fluorenyl Pendant Groups: Synthesis, Characterization, and Electro-Optical Properties
}

\author{
SHENG-HSIUNG YANG, HSING-CHUAN LI, CHIEN-KAI CHEN, CHAIN-SHU HSU \\ Department of Applied Chemistry, National Chiao Tung University, Hsinchu 30010, Taiwan, \\ Republic of China
}

Received 14 August 2006; accepted 5 September 2006

DOI: 10.1002 / pola. 21773

Published online in Wiley InterScience (www.interscience.wiley.com).

\begin{abstract}
Two series of poly(2,3-diphenyl-1,4-phenylenevinylene) (DP-PPV) derivatives containing multiple bulky substituents were synthesized. In the first series, two different groups were incorporated on C-5,6 positions of the phenylene moiety to increase steric hindrance and to obtain blue-shifted emissions. In the second series, bulky fluorenyl groups with two hexyl chains on the C-9 position were introduced on two phenyl pendants to increase the solubility as well as steric hindrance to prevent close packing of the main chain. Polymers with high molecular weights and fine-tuned electro-optical properties were obtained by controlling the feed ratio of different monomers during polymerization. The maximum photoluminescent emissions of the thin films are located between 384 and $541 \mathrm{~nm}$. Cyclic voltammetric analysis reveals that the band gaps of these light-emitting materials are in the range from 2.4 to $3.3 \mathrm{eV}$. A double-layer EL device with the configuration of ITO/PEDOT/P4/Ca/Al emitted pure green light with $\mathrm{CIE}^{\prime} 1931$ at $(0.24,0.5)$. Using copolymer P6 as the emissive layer, the maximum luminescence and current efficiency were both improved when compared with the homopolymer P4. () 2006 Wiley Periodicals, Inc. J Polym Sci Part A: Polym Chem 44: 6738-6749, 2006
\end{abstract}

Keywords: conjugated polymers; fluorescence; light-emitting diodes

\section{INTRODUCTION}

Semiconducting polymers have been intensively investigated for their potential applications in light-emitting diodes, ${ }^{1}$ thin film transitors, ${ }^{2}$ organic laser, ${ }^{3}$ and solar cells. ${ }^{4}$ Among them, poly (1,4-phenylene vinylene) (PPV) has attracted a great deal of attention in recent years because of its unique structure and highly electroluminescent (EL) properties. ${ }^{5}$ Long alkyl chains and/or

Correspondence to: C.-S. Hsu (E-mail: cshsu@mail.nctu. edu.tw)

Journal of Polymer Science: Part A: Polymer Chemistry, Vol. 44, 6738-6749 (2006) (O2006 Wiley Periodicals, Inc.

6738 bulky substitutents have been incorporated onto the PPV main chain to improve its solubility to cast thin films by solution process. Electron donating/withdrawing groups have also been introduced to adjust the optical and electrical properties. Until now many PPV derivatives have been synthesized to investigate their potential applications, for example, poly[2-methoxy-5-(2'ethylhexoxy)-1,4-phenylenevinylene] (MEH-PPV) is an orange-red emissive polymer and soluble in common organic solvents. ${ }^{6}$ Thin film of MEHPPV can be obtained from a spin-coating process. Cyano-substituted poly(2,5-dialkoxy-1,4-phenylene vinylene) (CN-PPV) is a red emissive polymer with high electron affinities. ${ }^{7,8}$ Silyl-substituted 
PPV is a greenish emissive material with a tendency to be easily charged by electrons rather than holes. ${ }^{9,10}$

Hsieh et al. first proposed a synthetic route to poly(2,3-diphenyl-1,4-phenylene vinylene) (DPPPV), which exhibits high photoluminescence (PL) efficiency in the solid state. ${ }^{11}$ Different substituents were introduced at C-5 position of the phenylene moiety to modify its properties. For example, highly phenylated DP-PPV was synthesized to further improve PL efficiency. ${ }^{12}$ Long alkyl chains were incorporated to improve the solubility of the polymer. ${ }^{13}$ Liquid crystalline side chains were also incorporated to achieve polarized emissions. ${ }^{14,15}$ By following this synthetic route, monomers containing diverse functional groups are easily synthesized and therefore soluble DP-PPV derivatives with high molecular weights are also easily obtained. Despite the advantages mentioned above, low device performance by using DP-PPVs as active layers is obtained and thus restricts their potential use for display applications. Recently, we have reported two series of DP-PPV derivatives containing long branched alkoxy and fluorenyl substituents. Both the brightness and current efficiency are highly improved. ${ }^{16}$

PPV, originally, is a yellow-green emissive polymer. Pure blue, green, and red emissions are not easy to achieve for fully conjugated PPV. It is generally thought that increase of steric hindrance can reduce the conjugation length of the polymer chain and tune the emission to blue light region. For this reason, many bulky groups have been incorporated and blue-shifted emissions were obtained. ${ }^{17,18} \mathrm{Hsieh}$ et al. ever investigated 2,3,5,6-tetraphenyl PPV in their synthetic route. However, they met the problem of reducing sterically hindered ester groups, and the predictably insoluble chlorine precursor polymer discouraged further investigation. ${ }^{12}$

In this study, two series of DP-PPV derivatives were designed to modify luminescent properties. In the first series, asymmetrically bulky substituents were incorporated on $\mathrm{C}-5,6$ positions of the phenylene moiety to further increase steric hindrance and to decrease the conjugation length of the main chain. The obtained DP-PPV derivatives were predicted to show blue-shifted emission. In the second series, a novel DP-PPV structure with two fluorenyl substituents on phenyl pendants was synthesized. This fluorenylphenyl group is essentially bulky to increase steric hindrance and to prevent close packing of polymer chains. Two hexyl chains at C-9 position can also help to increase the solubility. The homopolymer and two copolymers derived from copolymerizing with 1,4bis(chloromethyl)-2,5-dimethoxybenzene and 1,4bis(chloromethyl)-2-[4'-(3,7-dimethyloctoxy)phenyl]3 -phenylbenzene ${ }^{16}$ were also synthesized. The electrical and spectroscopic properties of these polymers were systematically investigated. In addition, double-layer light-emitting devices were also fabricated to study EL properties of the polymers.

\section{EXPERIMENTAL}

\section{Characterization Methods}

${ }^{1} \mathrm{H}$ NMR spectra were measured with a Varian 300 $\mathrm{MHz}$ spectrometer. Gel permeation chromatography data assembled from a Viscotek T50A Differential Viscometer and a LR125 Laser Refractometer and three columns in series were used to measure the molecular weights of polymers relative to polystyrene standards at $35{ }^{\circ} \mathrm{C}$. Differential scanning calorimetry (DSC) was performed on a PerkinElmer Pyris Diamond DSC instrument at a scan rate of $10{ }^{\circ} \mathrm{C} \mathrm{min}{ }^{-1}$. Thermal gravimetric analysis (TGA) was undertaken on a Perkin-Elmer Pyris 1 TGA instrument with a heating rate of $10{ }^{\circ} \mathrm{C}$ $\min ^{-1}$. UV-vis absorption spectra were obtained with an HP 8453 diode array spectrophotometer. PL emission spectra were obtained using ARC SpectraPro-150 luminescence spectrometer. Cyclic voltammetric $(\mathrm{CV})$ measurements were made in acetonitrile $\left(\mathrm{CH}_{3} \mathrm{CN}\right)$ with $0.1 \mathrm{M}$ tetrabutylammonium hexafluorophosphate $\left(\mathrm{TBAPF}_{6}\right)$ as the supporting electrolyte at a scan rate of $50 \mathrm{mV} / \mathrm{s}$. Platinum wires were used as both the counter and working electrodes, and silver/silver ions $(\mathrm{Ag}$ in $0.1 \mathrm{M} \mathrm{AgNO}_{3}$ solution, from Bioanalytical Systems, Inc.) as the reference electrode, and ferrocene was used as an internal standard. The corresponding highest-occupied molecular orbital (HOMO) and lowest-unoccupied molecular orbital (LUMO) energy levels were estimated from the onset redox potentials.

\section{Synthesis of Monomers M1-M4}

All reagents and chemicals were obtained from commercial sources (Aldrich, Merck, or TCI) and used without further purification. Tetrahydrofuran (THF) and dichloromethane $\left(\mathrm{CH}_{2} \mathrm{Cl}_{2}\right)$ were dried by distillation from sodium/benzophenone and calcium hydride, respectively. Schemes 1 and 

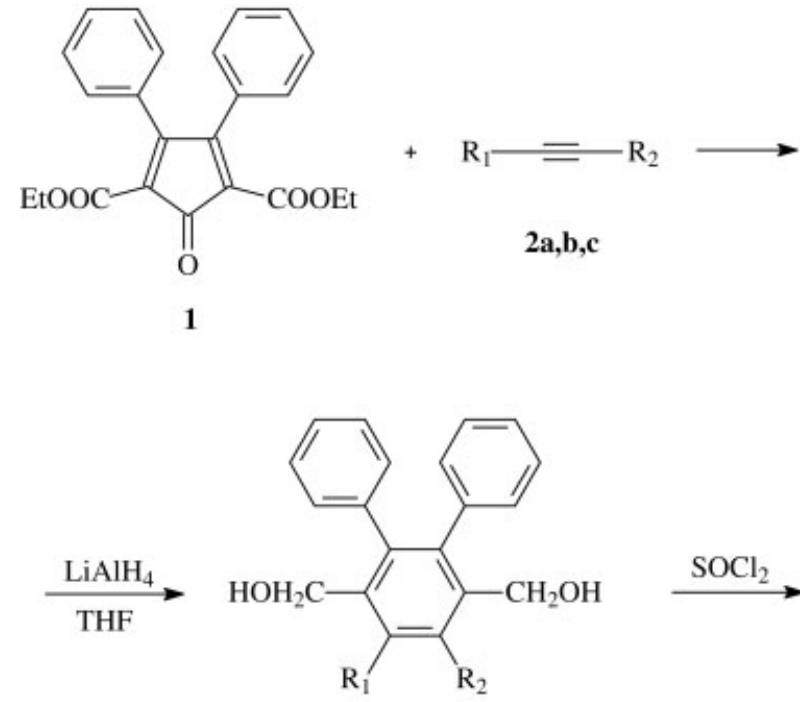

4a,b,c

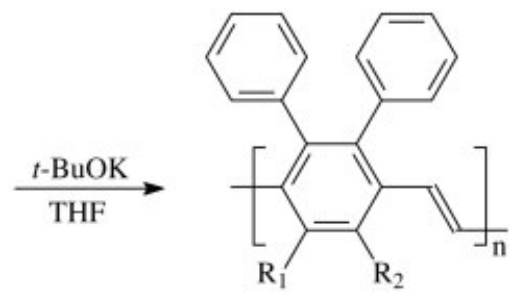

P1-P3<smiles>[R]c1c([R])c(C(=O)OCC)c(-c2ccccc2)c(-c2ccccc2)c1C(=O)OCC</smiles>

3a,b,c

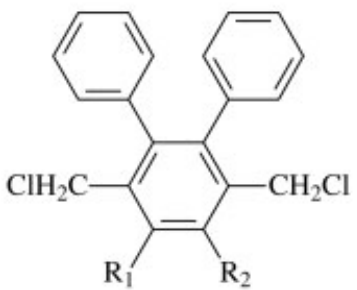

M1-M3

Scheme 1. Synthesis of polymers P1-P3.

2 outline the synthetic routes for monomers M1M4. The starting materials 2,5-dicarbethoxy-3,4diphenylcyclopentadienone, (1) 1-phenyl-1hexyne (2b), and 4,4'-dibromobenzil (5) were obtained from Aldrich Corp. The compound 2-(1-ethynyl)-9,9dihexylfluorene (2c) was synthesized as described previously in the literature. ${ }^{19}$

\section{1-(2-Ethylhexoxy)-4-(2-phenyl-1-ethynyl) benzene ( $2 a)$}

To a solution of phenylacetylene $(7.3 \mathrm{~g}, \quad 71.7$ $\mathrm{mmol})$ in triethylamine $(350 \mathrm{~mL})$ was added 1-(2ethylhexoxy)-4-iodobenzene (22.6 g, $68.1 \mathrm{mmol})$, triphenylphosphine ( $1.43 \mathrm{~g}, 5.46 \mathrm{mmol}), \mathrm{CuI}(0.54 \mathrm{~g}$, $2.87 \mathrm{mmol}$ ), and bis(triphenylphosphine) palladium(II) chloride $(0.48 \mathrm{~g}, 0.68 \mathrm{mmol})$. The mixture was refluxed at $85{ }^{\circ} \mathrm{C}$ for $12 \mathrm{~h}$. After cooling to room temperature, the crude product was filtered, washed with a large amount of $n$-hexane, and dried. It was purified by recrystallization from methanol to yield $17.7 \mathrm{~g}$ (85\%) of white crystals; $\mathrm{mp} 51{ }^{\circ} \mathrm{C}$.

${ }^{1} \mathrm{H} \mathrm{NMR}\left(\mathrm{CDCl}_{3}, \delta, \mathrm{ppm}\right): 0.9-0.96(\mathrm{t}, J=7 \mathrm{~Hz}$, $\left.6 \mathrm{H}, \quad-\mathrm{OCH}_{2}-\mathrm{CH}\left(\mathrm{CH}_{2} \mathrm{CH}_{3}\right)\left(\mathrm{CH}_{2}\right)_{3} \mathrm{CH}_{3}\right), \quad 1.28-$ $1.52\left(\mathrm{~m}, 8 \mathrm{H},-\mathrm{OCH}_{2}-\mathrm{CH}\left(\mathrm{CH}_{2} \mathrm{CH}_{3}\right)\left(\mathrm{CH}_{2}\right)_{3} \mathrm{CH}_{3}\right)$, $1.69\left(\mathrm{~m}, 1 \mathrm{H},-\mathrm{O}-\mathrm{CH}_{2}-\mathrm{CH}-\right), 3.78(\mathrm{~d}, J=6.4$ $\left.\mathrm{Hz}, 2 \mathrm{H},-\mathrm{OCH}_{2}-\right), 6.88(\mathrm{~d}, J=6.8 \mathrm{~Hz}, 4 \mathrm{H}$, $\left.-\mathrm{C} \equiv \mathrm{C}-\mathrm{C}_{6} \mathrm{H}_{4}-\mathrm{O}-\right), 7.33-7.57\left(\mathrm{~m}, 5 \mathrm{H}, \mathrm{C}_{6} \mathrm{H}_{5}\right.$ $-\mathrm{C} \equiv \mathrm{C}-$ ).

\section{Diethyl 2-(4'-(2-ethylhexoxy)phenyl)-3,5,6-triphenyl terephthalate (3a)}

To a $100 \mathrm{~mL}$ round flask was added a mixture of $\mathbf{1}$ $(9.72 \mathrm{~g}, 25.8 \mathrm{mmol})$ and $\mathbf{2 a}(3.0 \mathrm{~g}, 10.3 \mathrm{mmol})$ and refluxed at $170{ }^{\circ} \mathrm{C}$ for $12 \mathrm{~h}$. After cooling to room temperature, the crude product was purified by gel chromatography (silica gel, $n$-hexane as the eluent), following recrystallization from $n$-hexane to give $4.7 \mathrm{~g}(71 \%)$ of white crystals; $\mathrm{mp} 122{ }^{\circ} \mathrm{C}$.

${ }^{1} \mathrm{H} \mathrm{NMR}\left(\mathrm{CDCl}_{3}, \delta, \mathrm{ppm}\right): 0.58-0.66(\mathrm{~m}, 6 \mathrm{H}$, $\left.-\mathrm{CO}_{2}-\mathrm{CH}_{2}-\mathrm{CH}_{3}\right), 0.81$ (t, $J=7 \mathrm{~Hz}, 6 \mathrm{H}$, Journal of Polymer Science: Part A: Polymer Chemistry 

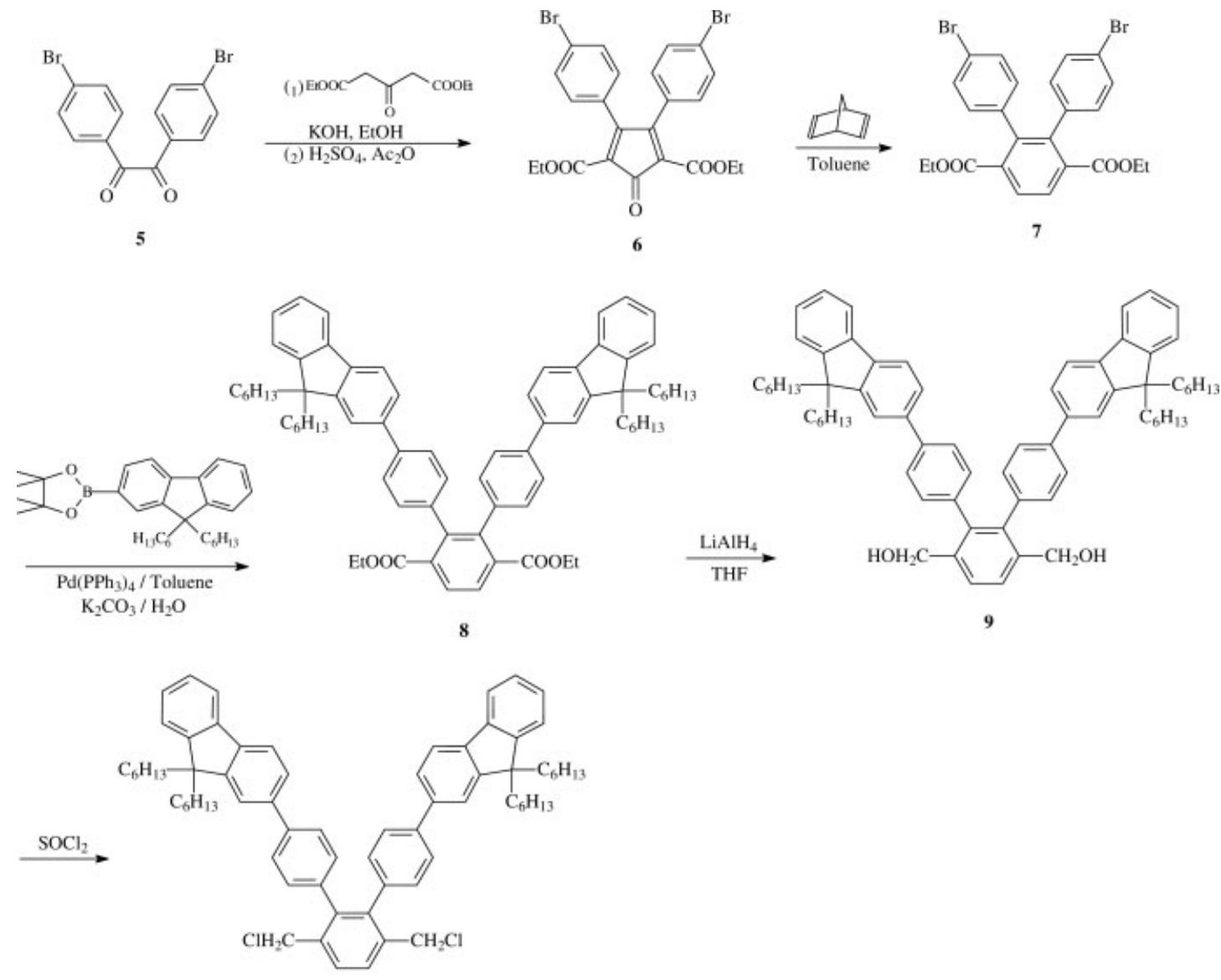

M4

Scheme 2. Synthesis of monomer M4.

$\left.-\mathrm{OCH}_{2}-\mathrm{CH}\left(\mathrm{CH}_{2} \mathrm{CH}_{3}\right)\left(\mathrm{CH}_{2}\right)_{3} \mathrm{CH}_{3}\right)$, 1.2-1.66 (m, $\left.9 \mathrm{H},-\mathrm{OCH}_{2}-\mathrm{CH}\left(\mathrm{CH}_{2} \mathrm{CH}_{3}\right)\left(\mathrm{CH}_{2}\right)_{3} \mathrm{CH}_{3}\right), 3.55-3.61$ $\left(\mathrm{m}, 4 \mathrm{H},-\mathrm{CO}_{2}-\mathrm{CH}_{2}-\right), 3.72(\mathrm{~d}, J=6.4 \mathrm{~Hz}, 2 \mathrm{H}$, $\left.-\mathrm{OCH}_{2}-\right), 6.57(\mathrm{~d}, J=7.2 \mathrm{~Hz}, 2 \mathrm{H}$, aromatic-H), $6.91(\mathrm{~d}, J=7.2 \mathrm{~Hz}, 2 \mathrm{H}$, aromatic-H), 7.03-7.08 (m, 15H, aromatic-H).

\section{Diethyl 2-butyl-3,5,6-triphenyl terephthalate (3b)}

By following the synthetic procedure for $\mathbf{3 a}$ and using $\mathbf{2 b}$ as starting material, the compound $\mathbf{3 b}$ was obtained as white crystals ( $61 \%$ yield); mp $133^{\circ} \mathrm{C}$.

${ }^{1} \mathrm{H}$ NMR $\left(\mathrm{CDCl}_{3}, \delta, \mathrm{ppm}\right): 0.61-0.67(\mathrm{t}, J=7$ $\left.\mathrm{Hz}, 3 \mathrm{H},-\left(\mathrm{CH}_{2}\right)_{3}-\mathrm{CH}_{3}\right), 0.87(\mathrm{t}, J=7 \mathrm{~Hz}, 6 \mathrm{H}$, $\left.-\mathrm{CO}_{2}-\mathrm{CH}_{2} \mathrm{CH}_{3}\right), 1.04\left(\mathrm{~m}, 2 \mathrm{H}\right.$, phenyl- $\mathrm{CH}_{2} \mathrm{CH}_{2}$ $\mathrm{CH}_{2} \mathrm{CH}_{3}$ ), 1.33-1.42 (m, 2H, phenyl- $\mathrm{CH}_{2} \mathrm{CH}_{2} \mathrm{CH}_{2}$ $\left.\mathrm{CH}_{3}\right), 2.43-2.45\left(\mathrm{~m}, 2 \mathrm{H}\right.$, phenyl- $\mathrm{CH}_{2} \mathrm{CH}_{2} \mathrm{CH}_{2}$ $\left.\mathrm{CH}_{3}\right), 3.51-3.94\left(\mathrm{~m}, 4 \mathrm{H},-\mathrm{CO}_{2}-\mathrm{CH}_{2}-\right), 7.01-$ $7.12(\mathrm{~m}, 10 \mathrm{H}$, aromatic-H), 7.29-7.37 (m, 5H, aromatic-H).

Journal of Polymer Science: Part A: Polymer Chemistry DOI 10.1002/pola
Diethyl 2-(9,9-dihexylfluoren-2-yl)-5,6-diphenyl terephthalate (3c)

By following the synthetic procedure for $\mathbf{3 a}$ and using $\mathbf{2 c}$ as starting material, the compound $\mathbf{3 c}$ was obtained as light-brown liquid ( $80 \%$ yield).

${ }^{1} \mathrm{H}$ NMR $\left(\mathrm{CDCl}_{3}, \delta, \mathrm{ppm}\right): 0.61-1.26(\mathrm{~m}, 28 \mathrm{H}$, fluorene- $\mathrm{CH}_{2}-\left(\mathrm{CH}_{2}\right)_{4}-\mathrm{CH}_{3}, \quad-\mathrm{CO}_{2}-\mathrm{CH}_{2}$ $\left.\mathrm{CH}_{3}\right), 1.82\left(\mathrm{t}, \mathrm{J}=6.8 \mathrm{~Hz}, 4 \mathrm{H}\right.$, fluorene- $\left.\mathrm{CH}_{2}-\right)$, 6.99-7.14 (m, 10H, aromatic-H), 7.32-7.34 (m, $3 \mathrm{H}$, aromatic-H), $7.44(\mathrm{~d}, J=7.4 \mathrm{~Hz}, 2 \mathrm{H}$, aromatic- $\mathrm{H}), 7.73(\mathrm{t}, J=7.8 \mathrm{~Hz}, 2 \mathrm{H}$, aromatic- $\mathrm{H})$, $7.86(\mathrm{~s}, 1 \mathrm{H}$, aromatic-H).

\section{1,4-Bis(hydroxymethyl)-2-(4' -(2-ethylhexoxy)- phenyl)-3,5,6-triphenylbenzene (4a)}

A solution of $\mathbf{3 a}(3.0 \mathrm{~g}, 4.7 \mathrm{mmol})$ in THF $(30 \mathrm{~mL})$ was added dropwise with stirring to a suspension of $\mathrm{LiAlH}_{4}(1.02 \mathrm{~g}, 26.8 \mathrm{~mol})$ in THF $(40 \mathrm{~mL})$ at $0{ }^{\circ} \mathrm{C}$ under nitrogen atmosphere. The solution was then refluxed at $70{ }^{\circ} \mathrm{C}$ for $4 \mathrm{~h}$. After the solution was cooled in an ice-bath, a saturated 
$\mathrm{Na}_{2} \mathrm{SO}_{4}$ aqueous solution was added dropwise until the solution became white. The white precipitate was removed by filtration. The crude product was isolated by evaporating the solvent and purified by recrystallization from $n$-hexane to yield $2.33 \mathrm{~g}(90 \%)$ of white crystals; $\mathrm{mp} 175{ }^{\circ} \mathrm{C}$.

${ }^{1} \mathrm{H} \mathrm{NMR}\left(\mathrm{CDCl}_{3}, \delta, \mathrm{ppm}\right): 0.83(\mathrm{t}, J=7 \mathrm{~Hz}, 3 \mathrm{H}$, $\left.-\mathrm{OCH}_{2}-\mathrm{CH}\left(\mathrm{CH}_{2} \mathrm{CH}_{3}\right)\left(\mathrm{CH}_{2}\right)_{3} \mathrm{CH}_{3}\right), 1.22-1.32(\mathrm{~m}$, $\left.9 \mathrm{H},-\mathrm{OCH}_{2}-\mathrm{CH}\left(\mathrm{CH}_{2} \mathrm{CH}_{3}\right)\left(\mathrm{CH}_{2}\right)_{3} \mathrm{CH}_{3}\right), 3.68(\mathrm{t}$, $\left.J=6.8 \mathrm{~Hz}, 2 \mathrm{H},-\mathrm{OCH}_{2}-\right), 4.11(\mathrm{~d}, J=6.4 \mathrm{~Hz}$, $\left.2 \mathrm{H},-\mathrm{CH}_{2} \mathrm{OH}\right), 6.62(\mathrm{~d}, J=7.2 \mathrm{~Hz}, 2 \mathrm{H}$, aromatic$\mathrm{H}), 6.95(\mathrm{~d}, J=7.2 \mathrm{~Hz}, 2 \mathrm{H}$, aromatic-H), 7.05-7.13 (m, 15H, aromatic-H).

\section{1,4-Bis(hydroxymethyl)-2-butyl-3,5,6-triphenyl- benzene (4b)}

By following the synthetic procedure for $\mathbf{4 a}$ and using $\mathbf{3 b}$ as starting material, the compound $\mathbf{4 b}$ was obtained as white crystals (62\% yield); mp $234{ }^{\circ} \mathrm{C}$.

${ }^{1} \mathrm{H} \mathrm{NMR}\left(\mathrm{CDCl}_{3}, \delta, \mathrm{ppm}\right): 0.68(\mathrm{t}, J=7 \mathrm{~Hz}, 3 \mathrm{H}$, - $\left.\left(\mathrm{CH}_{2}\right)_{3}-\mathrm{CH}_{3}\right)$, 1.11-1.41 (m, $4 \mathrm{H}$, phenyl- $\mathrm{CH}_{2}$ $\left.\mathrm{CH}_{2} \mathrm{CH}_{2} \mathrm{CH}_{3}\right), 2.54\left(\mathrm{~m}, 2 \mathrm{H}\right.$, phenyl- $\mathrm{CH}_{2}$ $\left.\mathrm{CH}_{2} \mathrm{CH}_{2} \mathrm{CH}_{3}\right), 4.23\left(\mathrm{~d}, J=7.4 \mathrm{~Hz}, 4 \mathrm{H},-\mathrm{CH}_{2} \mathrm{OH}\right)$, $7.02-7.15(\mathrm{~m}, 10 \mathrm{H}$, aromatic-H), 7.32-7.45 (m, $5 \mathrm{H}$, aromatic-H).

\section{1,4-Bis(hydroxymethyl)-2-(9,9-dihexylfluoren-2-yl)-} 5,6-diphenylbenzene (4c)

By following the synthetic procedure for $\mathbf{4 a}$ and using $3 \mathbf{c}$ as starting material, the compound $\mathbf{4 c}$ was obtained as white crystals ( $85 \%$ yield); $\mathrm{mp} 140{ }^{\circ} \mathrm{C}$.

${ }^{1} \mathrm{H}$ NMR $\left(\mathrm{CDCl}_{3}, \delta, \mathrm{ppm}\right): 0.64-1.11(\mathrm{~m}, 22 \mathrm{H}$, fluorene- $\left.\mathrm{CH}_{2}-\left(\mathrm{CH}_{2}\right)_{4}-\mathrm{CH}_{3}\right), 1.92(\mathrm{t}, \mathrm{J}=6.8 \mathrm{~Hz}$, $4 \mathrm{H}$, fluorene- $\left.\mathrm{CH}_{2}-\right), 4.41(\mathrm{t}, J=7 \mathrm{~Hz}, 4 \mathrm{H}$, $\left.-\mathrm{CH}_{2} \mathrm{OH}\right), 7.01-7.18(\mathrm{~m}, 10 \mathrm{H}$, aromatic-H), 7.28-7.35 (m, 3H, aromatic- $\mathrm{H}), 7.49-7.6(\mathrm{~m}, 3 \mathrm{H}$, aromatic- $\mathrm{H}), 7.7-7.78(\mathrm{~m}, 2 \mathrm{H}$, aromatic- $\mathrm{H})$.

\section{1,4-Bis(chloromethyl)-2-(4'-(2-ethylhexoxy)phenyl)- 3,5,6-triphenylbenzene (M1)}

To a solution of $4 \mathbf{a}(1.0 \mathrm{~g}, 1.56 \mathrm{mmol})$ in anhydrous $\mathrm{CH}_{2} \mathrm{Cl}_{2}(30 \mathrm{~mL})$ was slowly added $5 \mathrm{~mL}$ of thionyl chloride and stirred overnight under a nitrogen atmosphere. Water was then added dropwise into the solution to destroy excess thionyl chloride. The mixture was extracted with $10 \% \mathrm{NaHCO}_{3}(\mathrm{aq})$ and organic phase was concentrated in vacuo. The crude product was purified by recrystallization from $n$-hexane to give $0.5 \mathrm{~g}$ (46\%) of white crystals; $\mathrm{mp} 139{ }^{\circ} \mathrm{C}$.
${ }^{1} \mathrm{H} \mathrm{NMR}\left(\mathrm{CDCl}_{3}, \delta, \mathrm{ppm}\right): 0.85(\mathrm{t}, J=7 \mathrm{~Hz}, 6 \mathrm{H}$, $\left.-\mathrm{OCH}_{2}-\mathrm{CH}\left(\mathrm{CH}_{2} \mathrm{CH}_{3}\right)\left(\mathrm{CH}_{2}\right)_{3} \mathrm{CH}_{3}\right)$, 1.1-1.32 (m, $\left.9 \mathrm{H},-\mathrm{OCH}_{2}-\mathrm{CH}\left(\mathrm{CH}_{2} \mathrm{CH}_{3}\right)\left(\mathrm{CH}_{2}\right)_{3} \mathrm{CH}_{3}\right), 3.72(\mathrm{t}, \mathrm{J}$ $\left.=6.8 \mathrm{~Hz}, 2 \mathrm{H},-\mathrm{OCH}_{2}-\right), 4.1(\mathrm{~d}, J=6.4 \mathrm{~Hz}, 2 \mathrm{H}$, $\left.-\mathrm{CH}_{2} \mathrm{Cl}\right), 6.66(\mathrm{~d}, J=7.2 \mathrm{~Hz}, 2 \mathrm{H}$, aromatic-H), $7.03(\mathrm{~d}, J=7.2 \mathrm{~Hz}, 2 \mathrm{H}$, aromatic-H), 7.05-7.13 (m, $15 \mathrm{H}$, aromatic-H).

\section{1,4-Bis(chloromethyl)-2-butyl-3,5,6-triphenyl- benzene (M2)}

By following the synthetic procedure for M1 and using $\mathbf{4 b}$ as starting material, the compound M2 was obtained as white crystals ( $42 \%$ yield); $\mathrm{mp}$ $184^{\circ} \mathrm{C}$

${ }^{1} \mathrm{H} \mathrm{NMR}\left(\mathrm{CDCl}_{3}, \delta, \mathrm{ppm}\right): 0.69(\mathrm{t}, J=7 \mathrm{~Hz}, 3 \mathrm{H}$, $\left.-\left(\mathrm{CH}_{2}\right)_{3}-\mathrm{CH}_{3}\right), 1.13-1.45$ (m, $4 \mathrm{H}$, phenyl- $\mathrm{CH}_{2}$ $\mathrm{CH}_{2} \mathrm{CH}_{2} \mathrm{CH}_{3}$ ), 2.54 (m, 2H, phenyl- $\mathrm{CH}_{2} \mathrm{CH}_{2} \mathrm{CH}_{2}$ $\left.\mathrm{CH}_{3}\right), 4.02\left(\mathrm{~d}, J=7.4 \mathrm{~Hz}, 4 \mathrm{H},-\mathrm{CH}_{2} \mathrm{Cl}\right), 7.03-$ $7.17(\mathrm{~m}, 10 \mathrm{H}$, aromatic-H), 7.35-7.47 (m, 5H, aromatic-H).

\section{1,4-Bis(chloromethyl)-2-(9,9-dihexylfluoren-2-yl)- 5,6-diphenylbenzene (M3)}

By following the synthetic procedure for $\mathbf{M 1}$ and using 4c as starting material, the compound M3 was obtained as white crystals (53\% yield); $\mathrm{mp}$ $60{ }^{\circ} \mathrm{C}$.

${ }^{1} \mathrm{H}$ NMR $\left(\mathrm{CDCl}_{3}, \delta, \mathrm{ppm}\right): 0.65-1.02(\mathrm{~m}, 22 \mathrm{H}$, fluorene- $\left.\mathrm{CH}_{2}-\left(\mathrm{CH}_{2}\right)_{4}-\mathrm{CH}_{3}\right), 1.98(\mathrm{t}, J=6.8 \mathrm{~Hz}$, $4 \mathrm{H}$, fluorene- $\left.\mathrm{CH}_{2}-\right), 4.29(\mathrm{t}, J=7 \mathrm{~Hz}, 4 \mathrm{H}$, $\left.-\mathrm{CH}_{2} \mathrm{Cl}\right), 7.04-7.16(\mathrm{~m}, 10 \mathrm{H}$, aromatic-H), 7.29$7.35(\mathrm{~m}, 3 \mathrm{H}$, aromatic- $\mathrm{H}), 7.43-7.64(\mathrm{~m}, 3 \mathrm{H}$, aromatic-H), 7.72-7.78 (m, 2H, aromatic-H).

\section{Diethyl 4,5-bis(4'-bromophenyl)-2-oxo-3,5-cyclo- pentadiene-1,3-dicarboxylate (6)}

To a mixture of $\mathbf{5}$ (10 g, $26.04 \mathrm{mmol})$ and diethyl 1,3 -acetonedicarboxylate $(5.26 \mathrm{~g}, 26.04 \mathrm{mmol})$ in ethanol (100 mL), a solution of KOH (1.46 g, 26.1 $\mathrm{mmol})$ in ethanol $(20 \mathrm{~mL})$ was added and stirred at room temperature for $24 \mathrm{~h}$. The resultant yellow precipitate was filtered off and dried. $\mathrm{H}_{2} \mathrm{SO}_{4}$ was then added dropwise with stirring to slurry of the yellow precipitate in acetic anhydride $(50 \mathrm{~mL})$ until the solution became dark-red. After $30 \mathrm{~min}, 50 \mathrm{~g}$ of ice was added slowly to decompose excess acetic anhydride, and the crude product was collected and dried. It was purified by recrystallization from methanol to give $11.2 \mathrm{~g}(78 \%)$ of white crystals; mp $118^{\circ} \mathrm{C}$.

Journal of Polymer Science: Part A: Polymer Chemistry DOI 10.1002/pola 
${ }^{1} \mathrm{H}$ NMR $\left(\mathrm{CDCl}_{3}, \delta, \mathrm{ppm}\right): 1.43-1.47(\mathrm{t}, J=7$ $\left.\mathrm{Hz}, 6 \mathrm{H},-\mathrm{CO}_{2}-\mathrm{CH}_{2} \mathrm{CH}_{3}\right), 4.31-4.36$ (q, $J=7$ $\left.\mathrm{Hz}, 4 \mathrm{H},-\mathrm{CO}_{2}-\mathrm{CH}_{2}-\right), 7.58-7.62(\mathrm{~m}, 4 \mathrm{H}$, aromatic-H), 7.66-7.69 (m, 4H, aromatic- $\mathrm{H})$.

\section{Diethyl 2,3-bis(4'-bromophenyl)terephthalate (7)}

A mixture of 6 (5 g, $9.1 \mathrm{mmol}$ ) and norbornadiene $(4.4 \mathrm{~g}, 36.67 \mathrm{mmol})$ were dissolved in toluene $(100$ $\mathrm{mL}$ ). The solution was refluxed at $120^{\circ} \mathrm{C}$ for $12 \mathrm{~h}$. After cooling to room temperature, the solution was concentrated in vacuo to remove the solvent and crude product was purified by recrystallization from methanol to give $3.9 \mathrm{~g}(78 \%)$ of white crystals; $\mathrm{mp} 127^{\circ} \mathrm{C}$.

${ }^{1} \mathrm{H}$ NMR $\left(\mathrm{CDCl}_{3}, \delta, \mathrm{ppm}\right): 1.28-1.32(\mathrm{t}, J$ $\left.=7 \mathrm{~Hz}, 6 \mathrm{H},-\mathrm{CO}_{2}-\mathrm{CH}_{2} \mathrm{CH}_{3}\right), 4.21-4.27(\mathrm{q}, J$ $\left.=7 \mathrm{~Hz}, 4 \mathrm{H},-\mathrm{CO}_{2}-\mathrm{CH}_{2}-\right), 7.11-7.15(\mathrm{~m}, 4 \mathrm{H}$, aromatic-H), 7.28-7.31 (m, 4H, aromatic-H), 8.07 (s, 2H, aromatic-H).

Diethyl 2,3-bis(4'-(9,9-dihexylfluoren-2-yl)phenyl)terephthalate (8)

To a mixture of $\mathbf{7}$ (1.9 g, $3.57 \mathrm{mmol}$ ), 2-(4,4,5,5-tetramethyl-1,3,2-dioxaborolane-2-yl)-9,9-dihexylfluorene $(3.4 \mathrm{~g}, 7.16 \mathrm{mmol}),{ }^{20}$ tetrakis(triphenylphosphine)palladium (6.0 $\left.\mathrm{mg}, 5.3 \times 10^{-3} \mathrm{mmol}\right)$, $\mathrm{K}_{2} \mathrm{CO}_{3}(2.2 \mathrm{~g}, 15.92 \mathrm{mmol})$, and Aliquat 336 (0.4 $\mathrm{g}, 0.99 \mathrm{mmol}$ ) was added $15 \mathrm{~mL}$ of anhydrous toluene and $4 \mathrm{~mL}$ of de-ionized water. The resulting mixture was refluxed at $85{ }^{\circ} \mathrm{C}$ for 2 days under nitrogen atmosphere. After cooling to room temperature, the crude product was filtered, washed with a large amount of $n$-hexane, and dried. It was purified by gel chromatography (silica gel, $n$ hexane:ethyl acetate $=10: 1$ as the eluent) to give $1.1 \mathrm{~g}(53 \%)$ of pale-yellow liquid.

${ }^{1} \mathrm{H}$ NMR $\left(\mathrm{CDCl}_{3}, \delta, \mathrm{ppm}\right): 0.91-0.94(\mathrm{t}, J=7$ $\mathrm{Hz}, 12 \mathrm{H}$, fluorene- $\left.\left(\mathrm{CH}_{2}\right)_{5}-\mathrm{CH}_{3}\right), 1.28-1.32(\mathrm{t}, J$ $\left.=7 \mathrm{~Hz}, 6 \mathrm{H},-\mathrm{CO}_{2}-\mathrm{CH}_{2} \mathrm{CH}_{3}\right), 1.33-1.44(\mathrm{~m}$, $32 \mathrm{H}$, fluorene- $\left.\mathrm{CH}_{2}-\left(\mathrm{CH}_{2}\right)_{4}-\right), 2.56-2.84(\mathrm{~m}, 8 \mathrm{H}$, fluorene- $\left.\mathrm{CH}_{2}-\right), 4.21-4.27(\mathrm{q}, J=7 \mathrm{~Hz}, 4 \mathrm{H}$, $\left.-\mathrm{CO}_{2}-\mathrm{CH}_{2}-\right), 6.78-6.8(\mathrm{~m}, 4 \mathrm{H}$, aromatic- $\mathrm{H})$, 6.99-7.64 $(\mathrm{m}, 16 \mathrm{H}$, aromatic-H), 7.91-7.92 (m, $2 \mathrm{H}$, aromatic- $\mathrm{H}), 8.07$ (s, $2 \mathrm{H}$, aromatic- $\mathrm{H})$.

\section{1,4-Bis(hydroxymethyl)-2,3-bis(4'-(9,9-dihexylfluo- ren-2-yl)phenyl)benzene (9)}

By following the synthetic procedure for $\mathbf{4 a}$ and using $\mathbf{8}$ as starting material, the compound $\mathbf{9}$ was obtained as white viscous liquid (90\% yield).

Journal of Polymer Science: Part A: Polymer Chemistry DOI 10.1002/pola
${ }^{1} \mathrm{H}$ NMR $\left(\mathrm{CDCl}_{3}, \delta, \mathrm{ppm}\right): 0.91-0.94(\mathrm{t}, J=7$ $\mathrm{Hz}, 12 \mathrm{H}$, fluorene- $\left.\left(\mathrm{CH}_{2}\right)_{5}-\mathrm{CH}_{3}\right), 1.23-1.44(\mathrm{~m}$, $32 \mathrm{H}$, fluorene- $\left.\mathrm{CH}_{2}-\left(\mathrm{CH}_{2}\right)_{4}-\right), 2.56-2.84(\mathrm{~m}, 8 \mathrm{H}$, fluorene- $\left.\mathrm{CH}_{2}-\right), 4.95(\mathrm{~d}, J=7.4 \mathrm{~Hz}, 4 \mathrm{H}$, $\left.-\mathrm{CH}_{2} \mathrm{OH}\right), 6.81-6.84(\mathrm{~m}, 4 \mathrm{H}$, aromatic- $\mathrm{H})$, 6.99-7.64 (m, 18H, aromatic-H), 7.9-7.91 (m, $2 \mathrm{H}$, aromatic- $\mathrm{H})$.

\section{1,4-Bis(chloromethyl)-2,3-bis(4'-(9,9-dihexylfluo- ren-2-yl)phenyl)benzene (M4)}

By following the synthetic procedure for M1 and using $\mathbf{9}$ as starting material, the compound M4 was obtained as white viscous liquid (92\% yield).

${ }^{1} \mathrm{H} \mathrm{NMR}\left(\mathrm{CDCl}_{3}, \delta, \mathrm{ppm}\right): 0.91-0.94(\mathrm{t}, J=7 \mathrm{~Hz}$, $12 \mathrm{H}$, fluorene- $\left.\left(\mathrm{CH}_{2}\right)_{5}-\mathrm{CH}_{3}\right), 1.23-1.44(\mathrm{~m}, 32 \mathrm{H}$, fluorene- $\left.\mathrm{CH}_{2}-\left(\mathrm{CH}_{2}\right)_{4}-\right), 2.56-2.84(\mathrm{~m}, 8 \mathrm{H}$, fluorene- $\left.\mathrm{CH}_{2}-\right), 5.07\left(\mathrm{~d}, J=7.2 \mathrm{~Hz}, 4 \mathrm{H},-\mathrm{CH}_{2} \mathrm{Cl}\right)$, 6.81-6.84 (m, $4 \mathrm{H}$, aromatic-H), 6.99-7.64 $(\mathrm{m}, 18 \mathrm{H}$, aromatic-H), 7.9-7.91 (m, 2H, aromatic-H).

1,4-Bis(chloromethyl)-2,5-dimethoxybenzene (M5) and 1,4-Bis(chloromethyl) -2-[4'-(3,7-dimethyloctoxy)phenyl]-3-phenylbenzene (M6)

Monomers M5 and M6 were synthesized as described previously in the literatures. ${ }^{16,21}$

\section{Synthesis of Polymers: General Procedure}

Schemes 1 and 3 outline the syntheses of polymers P1-P6. An experimental procedure for the polymer P1 is given below. To a solution of the monomer M1 $(2.0 \mathrm{~g}, 4.68 \mathrm{mmol})$ in THF $(50 \mathrm{~mL})$, a solution of potassium tert-butoxide (tert-BuOK, 12 equiv) in THF (15 mL) was added. The resulting mixture was stirred at room temperature for $24 \mathrm{~h}$ under nitrogen atmosphere. A solution of 2,6-ditert-butylphenol (6 equiv) as end-capping agent in THF $(20 \mathrm{~mL})$ was then added and stirred for $6 \mathrm{~h}$. The polymer was obtained by pouring the mixture into methanol and filtered. It was purified by dissolving in THF and reprecipitated from methanol twice. After drying under vacuum for $24 \mathrm{~h}$, the polymer was obtained as brown solid $(0.55 \mathrm{~g}, 41 \%)$.

\section{Device Fabrication and Measurements}

Double-layer devices were fabricated as sandwich structures between calcium $(\mathrm{Ca})$ cathodes and indium-tin oxide (ITO) anodes. ITO-coated glass substrates were cleaned sequentially in ultrasonic baths of detergent, 2-propanol/deionized water (1:1 volume) mixture, toluene, de-ionized water, and ace- 

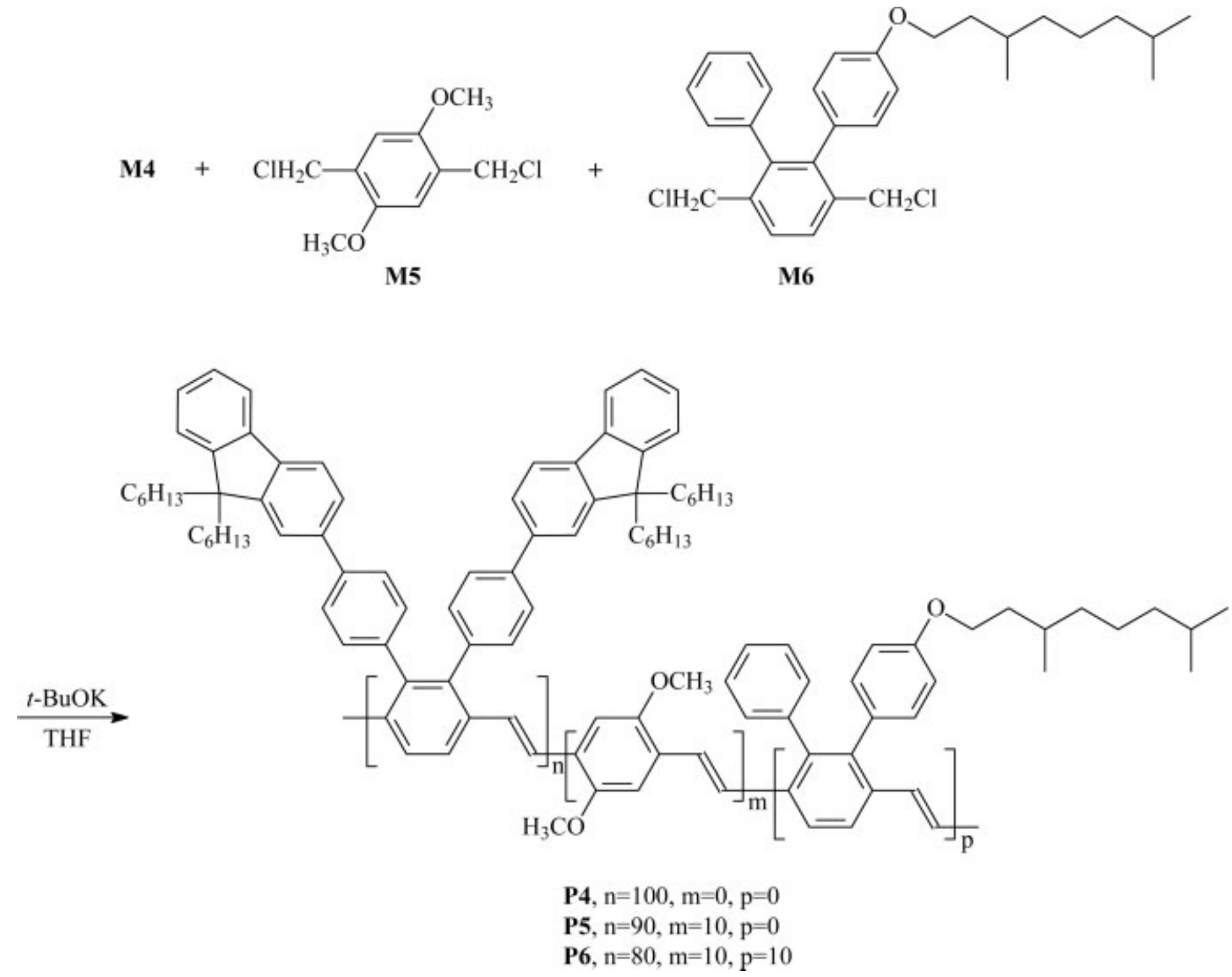

Scheme 3. Synthesis of polymers P4-P6.

tone. A $50 \mathrm{~nm}$ thick hole injection layer of poly(ethylenedioxythiophene) (PEDOT) doped with poly(styrenesulfonate) (PSS) was spin-coated on top of ITO from a $0.7 \mathrm{wt} \%$ dispersion in water and dried at $150{ }^{\circ} \mathrm{C}$ for $1 \mathrm{~h}$ in a vacuum. Thin films of synthesized polymers were spin-coated from toluene solutions onto the PEDOT layer and dried at $50{ }^{\circ} \mathrm{C}$ overnight in a vacuum. The thickness of the active layer was $\sim 50 \mathrm{~nm}$. Finally, $35 \mathrm{~nm} \mathrm{Ca}$ and $100 \mathrm{~nm} \mathrm{Al} \mathrm{elec-}$ trodes were made through a shadow mask onto the polymer films by thermal evaporation using an AUTO 306 vacuum coater (BOC Edwards, Wilmington, MA). Evaporations were carried out typically at base pressures lower than $2 \times 10^{-6}$ torr The active area of each EL device was $4 \mathrm{~mm}^{2}$ and the device was characterized following a published protocol. ${ }^{22}$

\section{RESULTS AND DISCUSSION}

\section{Synthesis of Polymers}

Scheme 1 outlines the synthetic route for monomers M1-M3. Different substituents of $R_{1}$ and $R_{2}$ were incorporated on the $\mathrm{C} 5$ and $\mathrm{C} 6$ positions of the phenylene moiety via the Diels-Alder reaction. The compound $\mathbf{4}$ with dihydroxy groups was obtained by the reduction of $\mathbf{3}$, following the chlorination to give monomers M1-M3. Scheme 2 outlines the synthesis of monomer M4. The synthetic route is similar to the Scheme 1, except the incorporation of two bulky fluorenyl groups via the Suzuki coupling reaction. Schemes 1 and 3 outline the syntheses of polymers P1-P6. The polymerization was carried out via a modified Gilch route to obtain soluble DP-PPV derivatives. For a typical Gilch synthetic route, $\alpha, \alpha^{\prime}$-dihalo- $p$-xylene is employed with excess amount of tert-BuOK in organic solvents. Alkyl or alkoxy chains are often incorporated on the aromatic rings to improve the solubility of the resulting polymers. The polymerization condition is mild and the molecular weights of obtained polymers are relatively large. In our experiments P1-P4 are homopolymers, while $\mathbf{P 5}$ and $\mathbf{P 6}$ are copolymers obtained from copolymerizing M4 with two different monomers M5 and M6. It has been reported that incorporation of $\mathbf{M 5}$ increased carrier mobility inside poly- 
Table 1. Feed Ratio and Polymerization Results of Polymers P1-P6

\begin{tabular}{rrrrrrrr}
\hline & \multicolumn{7}{c}{$\begin{array}{c}\text { Yield } \\
(\%)\end{array}$} \\
Polymer & $n$ & $m$ & $p$ & $\bar{M}_{n}$ & $\bar{M}_{w}$ & PDI \\
\hline P1 & 100 & & - & 33 & 1728 & 4888 & 2.59 \\
P2 & 100 & & - & 41 & 8882 & 41,025 & 4.61 \\
P3 & 100 & & - & 36 & 3695 & 6596 & 1.78 \\
P4 & 100 & & - & 55 & 580,800 & 557,000 & 1.15 \\
P5 & 90 & 10 & - & 58 & 556,200 & 600,300 & 1.08 \\
P6 & 80 & 10 & 10 & 54 & 332,900 & 360,000 & 1.08 \\
\hline
\end{tabular}

mer layer. ${ }^{23}$ M6 was first synthesized and reported by our group. The syntheses, characterization, and electro-optical properties of several DP-PPV derivatives based on M6 have been published previously. ${ }^{16}$ We expect that incorporation of $\mathbf{M 5}$ and M6 can adjust the optical and electrical properties of the homopolymer $\mathbf{P 4}$.

Table 1 summarizes the feed ratio of monomers, molecular weights, and polydispersity index (PDI) of resulting polymers. The number-average molecular weight $\bar{M}_{n}$ and weight-average molecular weights $\bar{M}_{w}$ of P1 and P3 are below $10^{4}$, regarding as oligomers. The $\bar{M}_{n}$ and $\bar{M}_{w}$ of $\mathbf{P 2}$ are 8882 and 41,025, respectively; however, its PDI value is large (4.61). The polymerization results for P1P3 are not quite satisfactory. For polymers P4P6, the $\bar{M}_{n}$ values are in the range from $3.3 \times 10^{5}$ to $5.8 \times 10^{5}$, while the $\bar{M}_{w}$ values are in the range from $3.6 \times 10^{5}$ to $6 \times 10^{5}$. The molecular weight distribution is relatively narrow (PDI $<1.2$ ). The obtained polymers P4-P6 are soluble in common organic solvents, such as chloroform, toluene, and chlorobenzene. Transparent and self-standing films can be cast from their solutions. Although two fluorenyl substituents are bulky, polymers with high molecular weights can still be obtained. However, the molecular weights of polymers P1-

Table 2. Thermal and Optical Properties of Polymers P1-P6

\begin{tabular}{|c|c|c|c|c|c|c|}
\hline \multirow[b]{2}{*}{ Polymer } & \multirow{2}{*}{$\begin{array}{c}T_{\mathrm{g}} \\
\left({ }^{\circ} \mathrm{C}\right)\end{array}$} & \multirow{2}{*}{$\begin{array}{c}T_{\mathrm{d}} \\
\left({ }^{\circ} \mathrm{C}\right)\end{array}$} & \multicolumn{2}{|c|}{ UV-vis (nm) } & \multicolumn{2}{|c|}{$\mathrm{PL}(\mathrm{nm})$} \\
\hline & & & Solution & Film & Solution & Film \\
\hline P1 & 166 & 330 & 297 & 303 & 407 & 384 \\
\hline P2 & 168 & 310 & 334 & 332 & 463 & 483 \\
\hline P3 & 160 & 433 & 389 & 390 & 500 & 506 \\
\hline P4 & 125 & 423 & 438 & 441 & 472 & 498 \\
\hline P5 & 144 & 421 & 449 & 452 & 505 & 535 \\
\hline P6 & 138 & 398 & 448 & 451 & 519 & 541 \\
\hline
\end{tabular}

Journal of Polymer Science: Part A: Polymer Chemistry DOI 10.1002/pola

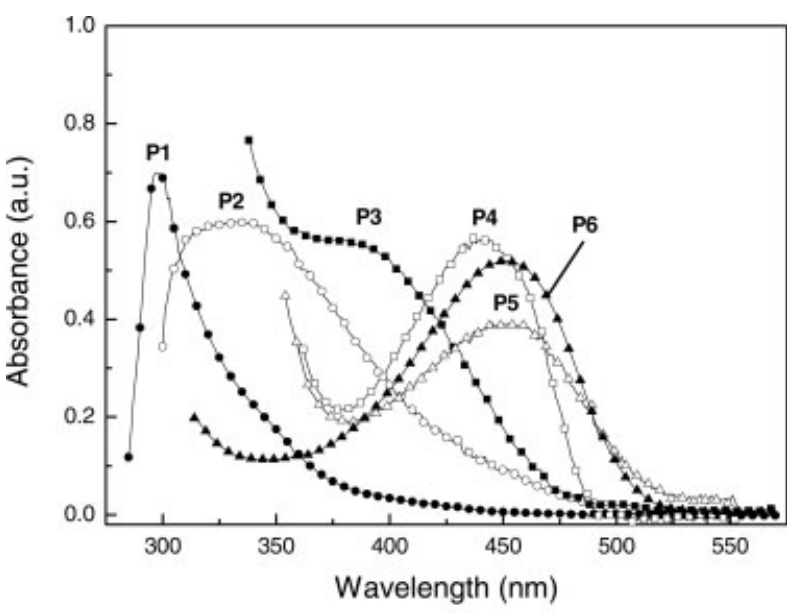

Figure 1. UV-vis absorption spectra of polymers P1P6 in thin film state.

P3 are much smaller. We explain this as a result of multiple substituents on the phenylene moiety. Monomers M1-M3 belong to tri- or tetra-substituted bis(chloromethyl)benzene, resulting in large steric hindrance. They are hindered to react with each other and hence polymers with low molecular weights and wide distribution were obtained. These materials show insufficient film-forming property that limits their potential use in thin-film devices. In contrast, monomers M4-M6 belong to disubstituted ones and the steric hindrance is smaller.

\section{Thermal Properties}

Table 2 summarizes the thermal properties of polymers P1-P6. Most polymers show good thermal

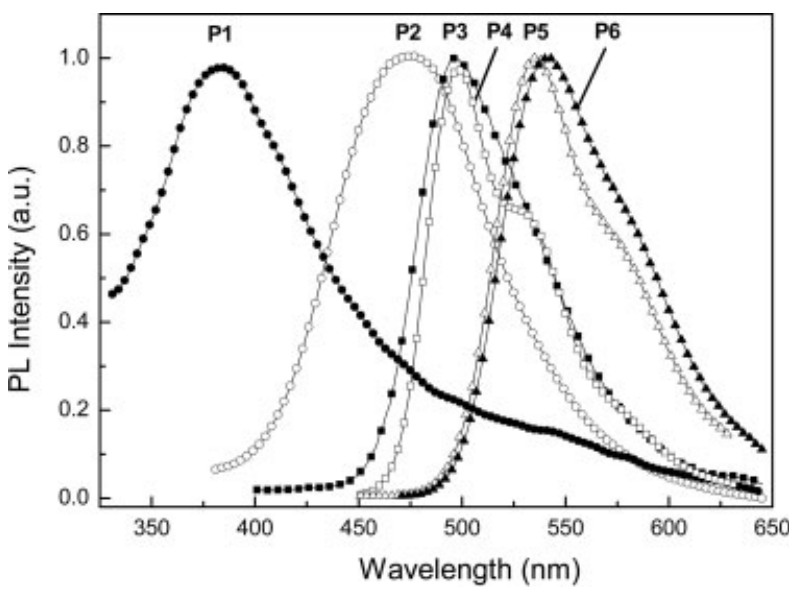

Figure 2. PL emission spectra of polymers P1-P6 in thin film state. 


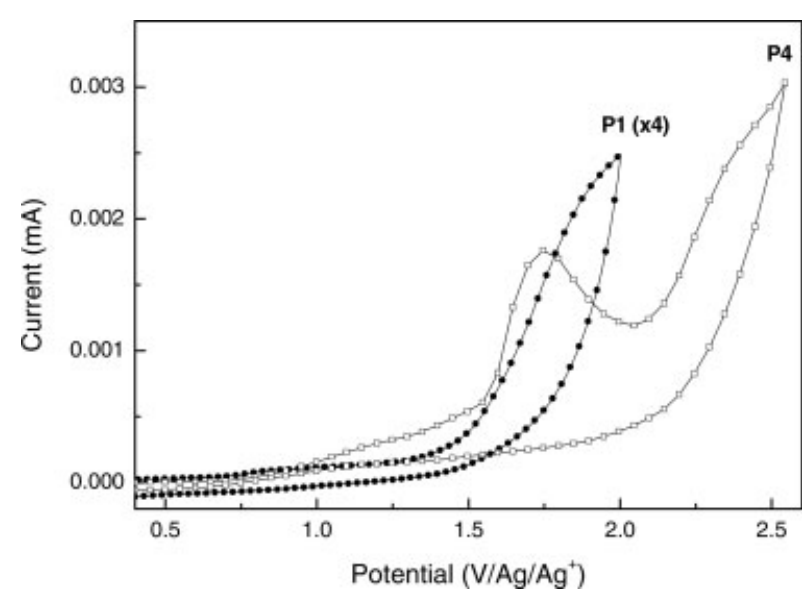

Figure 3. Cyclic voltammograms of homopolymers P1 and P4.

stabilities with high glass-transition temperatures $\left(T_{\mathrm{g}}\right)$ over $130{ }^{\circ} \mathrm{C}$ and high decomposition temperatures $\left(T_{\mathrm{d}}\right)$ over $310^{\circ} \mathrm{C}$. Polymers P3-P6 show even higher $T_{\mathrm{d}}$ than the others, which is attributed to high molecular weights and rigid fluorenyl group on the phenyl ring. On the other hand, P1 and P2 show lower $T_{\mathrm{d}}$ owing to low molecular weights; however, they show higher $T_{\mathrm{g}}$ as a result of tetrasubstitution on the phenylene moiety, which hinders the segmental motion of the main chain.

\section{Optical Properties}

Figure 1 shows the UV-vis absorption spectra of polymers P1-P6 in thin film state. Table 2 summarizes the UV-vis absorption maxima of all polymers in different states. The absorption maxima of synthesized polymers in toluene are located in the range from 297 to $449 \mathrm{~nm}$, which is attributed to the $\pi-\pi^{*}$ transition along the conjugated backbone. A small red-shift of the absorption band in thin film state is observed, which is due to the effect of inter- chain $\pi$-stacking. P1 shows the most blue-shifted absorption band because of lowest molecular weight, referring to the shortest conjugation length among six polymers. P3 shows more red-shifted absorption band than $\mathbf{P 1}$ and P2, implying a longer conjugation length. This is attributable to the steric effect of less substituents on the phenylene moiety, and a smaller torsional angle between two repeating units is formed. In contrast, copolymers P5 and P6 show similar absorption bands and are redshifted than P4, owing to two electron-donating alkoxy group on M5. It is also noted that incorporation of $\mathbf{M 6}$ does not affect the conjugation length significantly.

Figure 2 reveals the PL emission spectra of polymers P1-P6 in thin film state. The excitation wavelengths were based on their UV-vis absorption maxima in the same state. The PL emission maxima in different states are also summarized in Table 2. The maximum emission bands are located from 407 to $519 \mathrm{~nm}$ in solution state and from 384 to $541 \mathrm{~nm}$ in thin film state. Similar tendency of red-shift from solution to thin film state is observed, exclusive of $\mathbf{P 1}$. The emission maximum of $\mathbf{P 1}$ is located at $407 \mathrm{~nm}$ in toluene, while it is blue-shifted to $384 \mathrm{~nm}$ in film state. It is generally thought that red-shift in one state means some close packing of the polymer chains to extend the conjugation length. It seems that $\mathbf{P 1}$ in toluene has stronger $\pi-\pi$ interaction than in film state, may be due to similar structure of solvent and polymer structure. On the other hand, the shapes of PL spectra show some features. For P1-P3, no significant vibronic band is observed, indicating no or weak chain-chain interactions. The homopolymer $\mathbf{P} 4$ shows a clear emission shoulder band at $530 \mathrm{~nm}$, while the shoulder bands of copolymers P5 and P6 become less significant. This may be due to random structures of polymer main chains, which prevent close packing of neighboring polymers.

Table 3. Electrochemical Properties of Polymers P1-P6 in Solid Films

\begin{tabular}{cccccc}
\hline Polymer & $E_{\text {ox }}(\mathrm{V})$ & HOMO $(\mathrm{eV})$ & UV edge $(\mathrm{nm})$ & EG $(\mathrm{eV})$ & LUMO $(\mathrm{eV})$ \\
\hline P1 & 1.5 & -5.9 & 376 & 3.3 & -2.6 \\
P2 & 1.1 & -5.5 & 443 & 2.8 & -2.7 \\
P3 & 1.2 & -5.6 & 461 & 2.7 & -3.0 \\
P4 & 1.5 & -5.9 & 483 & 2.6 & -3.3 \\
P5 & 1.4 & -5.8 & 517 & 2.4 & -3.4 \\
P6 & 1.2 & -5.6 & 510 & 2.4 & -3.2 \\
\hline
\end{tabular}




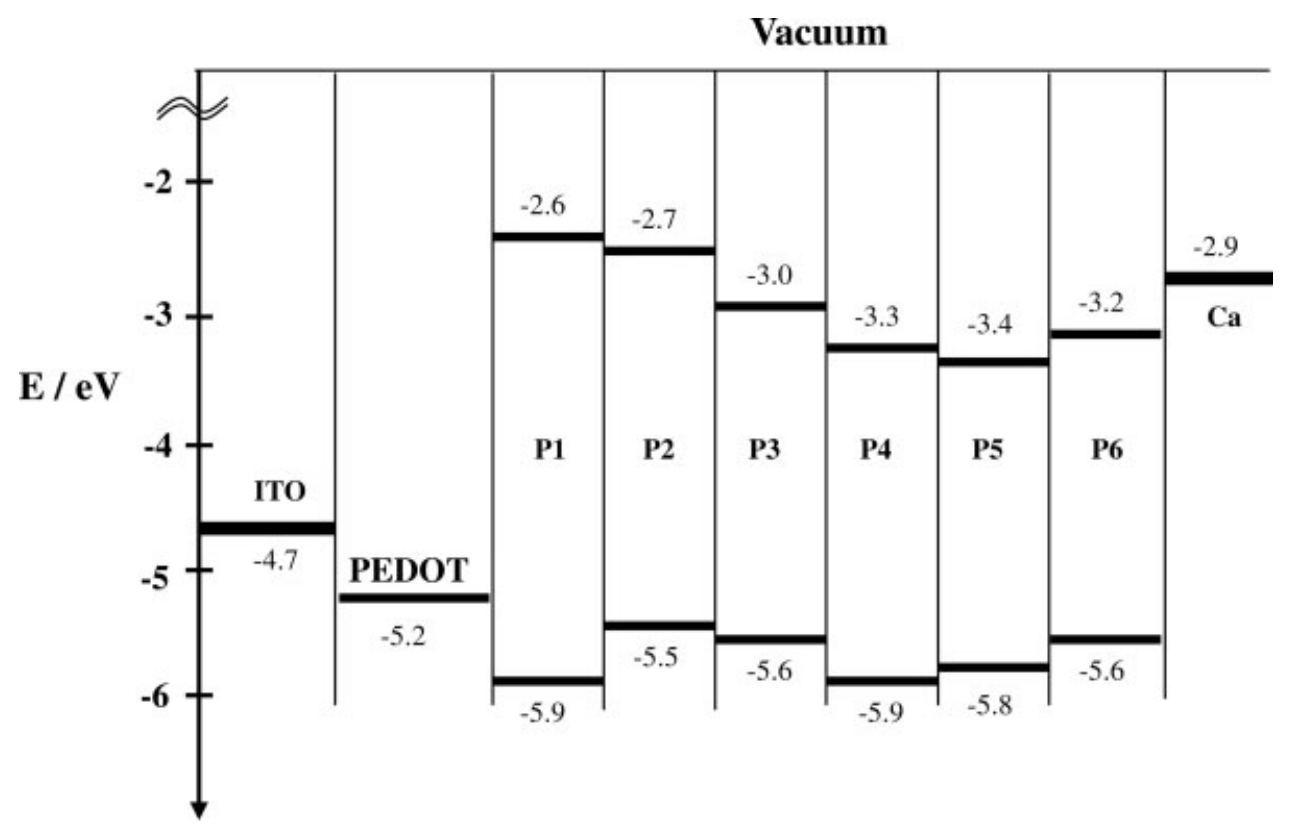

Figure 4. Energy level diagrams of polymers P1-P6.

\section{Electrochemical Analysis}

CV was employed to investigate the electrochemical behaviors of polymers synthesized and to estimate their energy levels. The oxidation process is clear and directly associated with the conjugation structure of the polymer. Figure 3 shows cyclic voltammograms of $\mathbf{P 1}$ and $\mathbf{P 4}$ in the oxidation process. The HOMO energy level is determined from the onset of the oxidation curve $\left(E_{\text {ox }}\right)$, which is given by

$$
\operatorname{HOMO}(\mathrm{eV})=-\left|E_{\text {ox }}+4.4\right|
$$

which is in the range from -5.9 to $-5.5 \mathrm{eV}$. The energy gaps (EG) of materials are determined from the edge of their UV-vis absorption spectra $\left(\lambda_{\text {onset }}\right)$, which is given by

$$
\mathrm{EG}(\mathrm{eV})=1240 / \lambda_{\text {onset }}
$$

which is in the range from 2.4 to $3.3 \mathrm{eV}$. Combining the electrochemical data and UV-vis charac- teristics give an estimate of the LUMO energy levels. Table 3 summarizes the HOMO, LUMO, and EG values of polymers P1-P6. The energy level diagram of these materials is illustrated in Figure 4. The relatively small $E_{\text {ox }}$ of $\mathbf{P 2}, \mathbf{P 3}$, and $\mathbf{P 6}$ reveals that they are favored for $p$-doping process when compared with others. The smaller energy barrier between polymer P2 (and P3, P6) and PEDOT layer also shows the benefit for hole injection. Turning to EA value, P3 and P6 show smaller energy barriers of $0.2 \mathrm{eV}$ to the cathode $\mathrm{Ca}$. This also implies that electron injection is favored for these two polymers.

\section{Device Performance}

Double-layer light-emitting diodes with the configuration of ITO/PEDOT/Polymer/Ca/Al were fabricated to evaluate the potential use of synthesized DP-PPV derivatives. Among them, P1-P3 has relatively low molecular weights and could

Table 4. Device Performance of Polymers P4-P6 in ITO/PEDOT/

\begin{tabular}{|c|c|c|c|c|c|c|}
\hline \multirow[b]{2}{*}{ Polymer } & \multirow{2}{*}{$\begin{array}{c}\mathrm{EL} \\
(\mathrm{nm})\end{array}$} & \multirow{2}{*}{$\begin{array}{l}V_{\text {turn-on }} \\
(\mathrm{V})\end{array}$} & \multirow{2}{*}{$\begin{array}{l}\text { Max. } \\
\text { Brightness } \\
\left(\mathrm{cd} / \mathrm{m}^{2}\right)\end{array}$} & \multirow{2}{*}{$\begin{array}{l}\text { Max. } \\
\text { Yield } \\
(\mathrm{cd} / \mathrm{A})\end{array}$} & \multicolumn{2}{|c|}{$\mathrm{CIE}^{\prime} 1931$} \\
\hline & & & & & $x$ & $y$ \\
\hline $\mathbf{P 4}$ & 492 & 6 & 492 & 0.11 & 0.24 & 0.5 \\
\hline P5 & 530 & 5 & 1184 & 0.14 & 0.37 & 0.58 \\
\hline P6 & 524 & 3 & 1298 & 0.22 & 0.34 & 0.58 \\
\hline
\end{tabular}
Polymer/Ca/Al Devices 
not provide sufficient film-forming properties. This may affect the device fabrication and performance evaluation. Hence, we fabricated three EL devices using $\mathbf{P 4 - P 6}$ as the active layer. The maximum EL emission bands of P4-P6 are listed in Table 4, which are located at 492, 530, and 524 $\mathrm{nm}$, respectively. The CIE coordinates of the three polymers are also shown in Table 4, referring to pure green (P4) and yellowish green (P5, P6). Some extent of blue-shift of the EL emission with increasing the operation voltage is also observed. This phenomenon has been observed previously and explained as a result of charge-trapping/ defect site from some head-to-head isomers. ${ }^{16}$

Figures 5 and 6 show the brightness-voltage and efficiency-current density characteristics, respectively, of the three devices fabricated using P4-P6 as the active layer. The device using homopolymer P4 as active layer showed a maximum brightness of $492 \mathrm{~cd} / \mathrm{m}^{2}$ at $10 \mathrm{~V}$, and a maximum current efficiency of $0.11 \mathrm{~cd} / \mathrm{A}$ at $8 \mathrm{~V}$. Using copolymer P5 as active layer, the device showed an improved brightness of $1184 \mathrm{~cd} / \mathrm{m}^{2}$ and current efficiency of $0.14 \mathrm{~cd} / \mathrm{A}$ at $10 \mathrm{~V}$. The luminescence is roughly improved by incorporating 2,5 -dimethoxy-1,4-phenylene vinylene unit. The device of P6 showed even higher brightness and current efficiency, which was 2-fold higher than the homopolymer P4. The better performance of devices using P5 and P6 as active layer is explained by the smaller energy barrier between metals and emissive layer. Holes/electrons are easier to be injected into copolymers P5 and P6 than the homopolymer P4, and thus higher opportunity of charge recombination occurs (Fig. 4). Moreover, the charge mobility may be somewhat improved

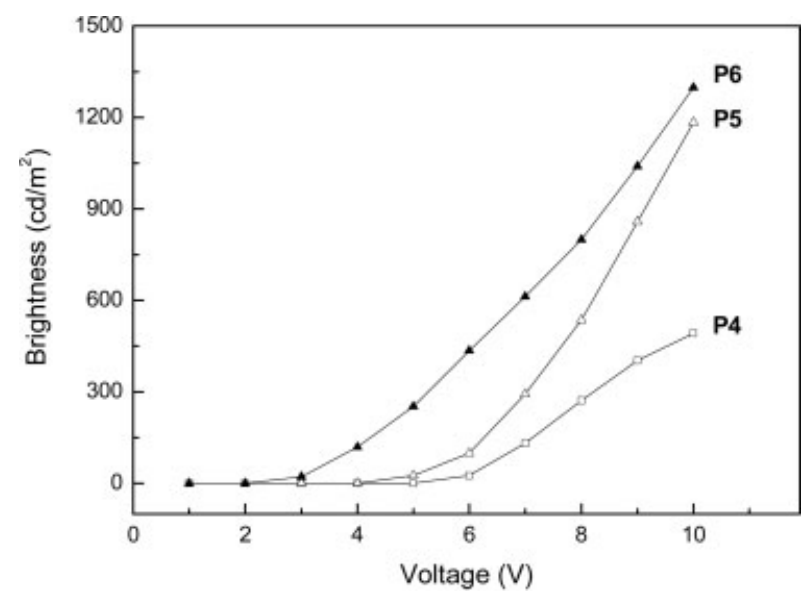

Figure 5. Brightness-voltage characteristics of polymers P4-P6 in ITO/PEDOT/Polymer/Ca/Al devices.

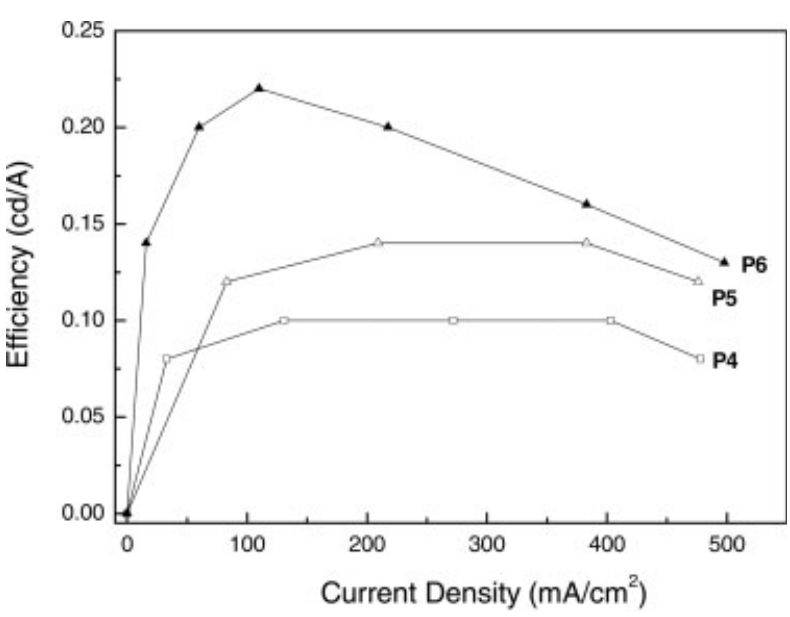

Figure 6. Efficiency-current density characteristics of polymers P4-P6 in ITO/PEDOT/Polymer/Ca/Al devices.

by incorporating $\mathbf{M 5}$ and $\mathbf{M 6}$. The device performance of these DP-PPV derivatives is summarized in Table 4. Compared with previous reports, the materials synthesized in this work showed moderate EL properties. Better device performance was obtained when compared with previous trisubstituted DP-PPVs, but not as high as those with one fluorenyl ring as pendant group. ${ }^{15,16} \mathrm{We}$ consider that two bulky substituents on the main chain may also hinder carrier transport and movement inside polymer layer.

\section{CONCLUSIONS}

In this work, six DP-PPV derivatives containing bulky substituents were synthesized. Highly phenylated DP-PPV derivatives with blue-shifted emission were obtained. However, the molecular weights are not very high. Bulky fluorenyl substituents with two hexyl chains were introduced on the pendant phenyl ring to increase steric hindrance and to prevent close packing of the main chains. The molecular weights of difluorenyl substituted DP-PPVs are sufficiently high. Doublelayer devices with the configuration of ITO/ $\mathrm{PEDOT} /$ polymer/Ca/Al were fabricated and characterized. Pure green light with $\mathrm{CIE}^{\prime} 1931$ at $(0.24,0.5)$ was observed for P4. Using $\mathbf{P 6}$ as the emissive layer, the maximum luminescence and current efficiency were both improved.

The authors thank the National Science Council (NSC) of the Republic of China (NSC 94-2120M-009-009) for financial support of this research.

Journal of Polymer Science: Part A: Polymer Chemistry DOI 10.1002/pola 


\section{REFERENCES AND NOTES}

1. Burroughes, J. H.; Bradley, D. D. C.; Brown, A. R.; Marks, R. N.; Mackay, K.; Friend, R. H.; Burn, P. L.; Holmes, A. B. Nature 1990, 347, 539.

2. Katz, H. E. J Mater Chem 1997, 7, 369.

3. Gustatsson, G.; Gao, Y.; Treacy, G. M.; Klavetter, F.; Colaneri, N.; Hegger, A. J. Nature 1992, 357, 47.

4. Chen, S. N.; Heeger, A. J.; Kiss, Z.; MacDiarmid, A. G.; Gau, S. C.; Peebles, D. L. Appl Phy Lett 1980, 36, 96.

5. Woodruff, M. Synth Met 1996, 80, 257.

6. Braun, D.; Heeger, A. J. Appl Phys Lett 1991, 58, 1982.

7. Greenham, N. C.; Moratti, S. C.; Bradley, D. D. C.; Friend, R. H.; Holmes, A. B. Nature 1993, 365, 628.

8. Chen, S. A.; Chang, E. C. Macromolecules 1998, 31, 4899.

9. Wang, L. H.; Chen, Z. K.; Kang, E. T.; Meng, H.; Huang, W. Synth Met 1999, 105, 85.

10. Chen, Z. K.; Huang, W.; Wang, L. H.; Kang, E. T.; Chen, B. J.; Lee, C. S.; Lee, S. T. Macromolecules 2000, 33, 9015.

11. Wan, W. C.; Antoniadis, H.; Choong, V. E.; Razafitrimo, H.; Gao, Y.; Field, W. A.; Hsieh, B. R. Macromolecules 1997, 30, 6567.
12. Hsieh, B. R.; Wan, W. C.; Yu, Y.; Gao, Y.; Goodwin, T. E.; Gonzalez, S. A.; Feld, W. A. Macromolecules 1998, 31, 631.

13. Hsieh, B. R.; Yu, Y.; Forsythe, E. W.; Schaaf, G. M.; Feld, W. A. J Am Chem Soc 1998, 120, 231.

14. Li, A. K.; Yang, S. S.; Jean, W. Y.; Hsu, C. S.; Hsieh, B. R. Chem Mater 2000, 12, 2741.

15. Yang, S. H.; Chen, J. T.; Li, A. K.; Huang, C. H.; Chen, K. B.; Hsieh, B. R.; Hsu, C. S. Thin Solid Films 2005, 477, 73.

16. Chen, K. B.; Li, H. C.; Chen, C. K.; Yang, S. H.; Hsieh, B. R.; Hsu, C. S. Macromolecules 2005, 38, 8617.

17. Peng, Z.; Zhang, J.; Xu, B. Macromolecules 1999, 32,5162

18. Johansson, D. M.; Theander, M.; Srdanov, G.; Yu, G.; Inganäs, O.; Andersson, M. R. Macromolecules 2001, 34,3716 .

19. Lee, S. H.; Nakamura, T.; Tsutsui, T. Org Lett 2005, 2001, 3 .

20. Geng, Y.; Chen, A. C. A.; Ou, J. J.; Chen, S. H. Chem Mater 2003, 15, 4352.

21. Antoun, S.; Karasz, F. E.; Lenz, R. W. J Polym Sci Part A: Polym Chem 1988, 26, 1809.

22. Chen, C. H.; Tang, C. W. Appl Phys Lett 2001, 79, 3711.

23. Yu, L. S.; Chen, S. A. Synth Met 2002, 132, 81. 